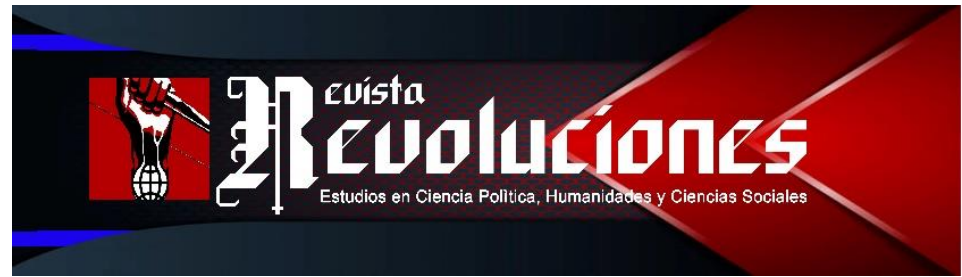

\title{
NO ME DEJES EN VISTO: USO DE DISPOSITIVOS MÓVILES EN LA ENSEÑNANZA DEL INGLÉS
}

\author{
Do not leave seen: the use of mobile devices in English teaching
}

\author{
Katherin Johanna Rodríguez- \\ Silva1 \\ UNIVERSIDAD SANTO TOMÁS \\ COLOMBIA \\ katherinrodriguez@usantotomas.edu.co \\ http://orcid.org/oooo-0002-7711-6832
}

\author{
Herberth Alfonso Mendieta- \\ Ramírez $^{2}$ \\ UNIVERSIDAD SANTO TOMÁS \\ COLOMBIA \\ herberthmendieta@usantotomas.edu.co \\ http://orcid.org/oooo-0002-1631-5416
}

DOI: https://doi.org/10.35622/j.rr.2020.02.004

Recibido: 02-I-2020 / Aceptado: 28-VI-2020 / Actualizado: 21-I-2021

\section{Resumen}

Este estudio de caso cualitativo y descriptivo tiene como objetivo analizar el efecto de la implementación de estrategias de aprendizaje móvil para desarrollar habilidades comunicativas en estudiantes de inglés de la Universidad Santo Tomás. Para lograrlo, se definieron tres momentos: el primero fue caracterizar algunas herramientas de aprendizaje móvil como aplicaciones de software enfocadas al desarrollo de habilidades en una lengua extranjera con el fin de identificar sus aportes al proceso de enseñanza. El segundo momento fue implementar una estrategia de aprendizaje móvil para verificar tanto el uso de las TIC como el efecto en el proceso académico. El último momento llevó al análisis de información para determinar los hallazgos y la comunicación de conclusiones. Esta investigación contó con el apoyo de 258 estudiantes, cuyo nivel de inglés osciló entre A1 y B1 según el MCER. Los resultados mostraron que los estudiantes se sintieron motivados para aprender y tuvieron un mejor desempeño con la mediación de estrategias y herramientas de aprendizaje móvil. Esta investigación contribuyó a la consolidación de una propuesta TIC para el Instituto de Idiomas de la Universidad.

Palabras Clave: Aprendizaje móvil, TIC, didáctica, metodología, enseñanza del inglés.

\footnotetext{
${ }^{1}$ Licenciada en Español-Inglés de la Universidad Pedagógica Nacional y Magister en Educación de la Universidad Santo Tomás. Se desempeña como investigadora y docente de inglés como lengua extranjera. Actualmente desarrolla procesos de enseñanza del inglés como lengua extranjera a nivel de posgrado como de evaluación e inclusión de TIC en el aula en pregrado.

${ }^{2}$ Licenciado en Español-Inglés de la Universidad Pedagógica Nacional y Magister en Educación de la Universidad Santo Tomás. Se desempeña como investigador y docente de inglés como lengua extranjera. Actualmente trabaja con temas relacionados con el currículo y la evaluación. También apoya procesos de calidad, acreditación y capacitación docente en educación superior.
} 


\begin{abstract}
This qualitative and descriptive case study aims to analyze the effect of the implementation of mobile learning strategies to develop communicative skills in English students at the Universidad Santo Tomas. To achieve this, three important moments were defined: the first moment was to characterize some mobile learning tools such as software applications focused on the development of skills in a foreign language in order to identify their contributions to the teaching process. The second moment was to implement a mobile learning strategy to verify both: the use of ICT and the effect in the academic process. The last moment led to the analysis of information to determine the findings and the communication of conclusions. To carry out this research, it was necessary the support of 258 students, whose English level ranged between $\mathrm{A} 1$ and $\mathrm{B} 1$ according to the CEFR. The results showed that students felt motivated to learn and they had a better performance with the mediation of mobile learning strategies and tools. This research contributed to the consolidation of an ICT proposal for the Languages Institute at the university.
\end{abstract}

Keyword: Mobile learning, ICT, didactics, methodology, teaching english.

\title{
INTRODUCCIÓN
}

Nuestra generación y aquella que tenemos en nuestras aulas son la generación de las comunicaciones. Hoy en día es fuera de lo común salir de casa sin el teléfono móvil, reproductor de música o computador portátil. Más inusual es pasar el día sin revisar los servicios de mensajería celular como Whatsapp o iMessage. Permanecemos en continua comunicación con el mundo, a través de las redes sociales o el correo electrónico, leemos las noticias desde los portales web de los noticieros y actualizamos permanente nuestro estado en Facebook. Nos comunicamos y también lo hacen nuestros estudiantes.

Algunos más osados que otros, los estudiantes en nuestras aulas organizan vía chat la hora de encuentro para el almuerzo, planifican la ruta de transporte que van a utilizar después de salir de la clase utilizando aplicaciones basadas en tecnología GPS, distribuyen las responsabilidades para la próxima presentación grupal en documentos publicados en un drive de información, corroboran lo que el profesor les presenta en la lección en un motor de búsqueda. Ellos, mejor que nosotros, permanecen conectados, están en línea.

En Colombia (hasta donde sabemos) existe una expresión que se usa cuando se envía un mensaje de texto, pero no se recibe respuesta: "Me dejaste en visto". Ahora con las nuevas actualizaciones es posible conocer la hora en que el receptor del mensaje leyó lo enviado lo que hace más difícil ignorar un mensaje no deseado. La experiencia que se presenta en la sistematización "No me dejes en visto" nace precisamente de la necesidad de incluir estrategias didácticas que permitieran la conexión con estudiantes que ya se encuentran conectados con sus dispositivos móviles. Es un intento de no enviar mensajes destinados a ser ignorados.

Revista Revoluciones -46- Vol. 2, No 2 (2020), pp. 45-59

Esta obra está bajo una licencia internacional Creative Commons Atribución 4.0. 
Dentro de la labor docente, de manera permanente se presenta el reto de actualizar los saberes propios de la disciplina, renovar los ejercicios didácticos en el aula, y estar a la vanguardia de los intereses de nuestros estudiantes. En este sentido, el presente capítulo expone el proceso que se ha llevado a cabo en la Universidad Santo Tomás de Bogotá, Colombia, en cuanto a la implementación de estrategias didácticas que hacen uso de dispositivos móviles, como herramienta innovadora dentro de los procesos de enseñanza de una segunda lengua, para este caso, el inglés.

Para lo anterior, el presente documento estará dividido en tres momentos. En el primero se realizará una breve contextualización de la inmersión de las tecnologías de la información y la comunicación en el ámbito educativo, para ello, retomaremos el concepto de globalización y sociedad del conocimiento para así llegar a considerar los retos que éste fenómeno trae para el sistema educativo. En segundo lugar, se llevará a cabo el análisis del estado actual de las competencias en segunda lengua en el sistema educativo colombiano, que justifica el presente proceso investigativo, al determinarse que, uno de los retos que se tienen en materia educativa en Colombia, es mejorar las competencias de aprendizaje en una segunda lengua; finalmente, con el contexto anterior, se mostrará el ejercicio investigativo desarrollado, en donde se verá cómo el uso de dispositivos móviles, dentro de los procesos formativos del idioma inglés, permite mejorar las competencias y alcanzar el aprendizaje significativo en los estudiantes.

Con todo lo anterior, se podrá establecer la necesidad de seguir involucrando en el ejercicio docente, diferentes tecnologías de la información y la comunicación dentro del proceso de enseñanza y aprendizaje de una segunda lengua que, como se verá, supone dos retos: primero por parte de los docentes que, a pesar de ser migrantes digitales deberán estar a la vanguardia de los intereses de los estudiantes; y en segundo lugar por parte de los estudiantes quienes también podrán encontrar nuevas formas de uso de los dispositivos que utilizan de manera cotidiana y que, con esta propuesta, se espera que se conviertan en elementos presentes en su desarrollo académico.

\section{DESARROLLO}

\section{Globalización y revolución tecnológica}

Para iniciar, hagamos una contextualización general de la globalización que "es, según sus acérrimos defensores, la creación de un espacio mundial de intercambio económico, productivo, financiero, político, ideológico y cultural, pero que bajo la nueva terminología se oculta la vieja aspiración del capital: la producción y el crecimiento económica a cuesta de lo que sea." (Salguero, 2011, p. 1).

En este sentido puede explicarse que la globalización busca el beneficio económico y monetario propio del modelo capitalista, que tiene a la vez efectos en todos los ámbitos de la vida humana, iniciando con el económico y financiero con sus efectos dominadores 
ampliamente estudiados; en referencia al ámbito cultural, se puede evidenciar una tendencia hacia la uniformidad, "todo esto posibilita la configuración de un modelo cultural o estilo de vida que crea las condiciones para una mayor dominación - domesticación ideológicocultural, instancia mediatizadora por excelencia para introyectar los valores afines al modelo económico neoliberal" (Ander Egg, 2000, p. 151) Así, se muestra al capitalismo como el estilo de vida ideal que se rige bajo la triada: dinero - consumo - status.

El tercer ámbito en el que el fenómeno de la globalización está presente, es el político el cual trasciende todos los demás aspectos de las naciones (cultura, política, educación, etc.), teniendo como base el compromiso de las naciones por el fomento a la competitividad y calidad en los procesos de bienes y servicios. Las repercusiones de esta saturación del estilo de vida capitalista alcanzan el aspecto psicológico a nivel individual y trasciende al ámbito de la cultura. Los valores culturales se han ido modificando, dando prevalencia a valores como el consumir, tener, ganar y competir.

En este sentido, hay una tendencia hacia la homogeneización cultural, que puede verse claramente en la uniformidad de gustos, de estilos de vida y comportamientos. La "americanización" del estilo de vida de los colombianos es evidente. Llama la atención la creciente necesidad de consumo del ciudadano de a pie, la celebración de fiestas que no son propias (por ejemplo, San Valentín) y la generalización por alcanzar el famoso "sueño americano". Ahora bien, en cuanto al impacto de este estilo de vida marcadamente consumista está la hiperconectividad en donde debemos detenernos por un momento.

La facilidad de acceso a la red hace que cada vez el mundo sea más pequeño. Las clases de Geografía solían tener como protagonistas los viejos afiches con los mapas del mundo y filminas con fotografías de lugares exóticos. Ahora con Google Maps, visitas virtuales y las fotografías panorámicas, esas culturas en otro tiempo lejanas están disponibles a la distancia de un clic. Y la posibilidad para un ciudadano de clase media de visitar aquellos lugares es más factible que nunca antes. Los gobiernos miden el alcance de las políticas educativas de las naciones en términos de conectividad y disponibilidad de servicios tecnológicos en el aula. En el caso colombiano, el Plan Nacional de Desarrollo 2010- 2014 a través del artículo 149 de la ley 1450 de 2011, Conectividad en Establecimientos Educativos, promueve el programa Conexión Total enfocado a la capacitación en uso de las TIC, ampliación de la cobertura en formación digital y de los recursos tecnológicos disponibles en los establecimientos educativos.

Programas como este han permitido que el acceso a las tecnologías de la información y la formación y el aprovechamiento de las mismas sean un elemento presente en la escuela colombiana. El impacto de la normatividad ha alcanzado cada vez una mayor población; como se muestra en el gráfico publicado por el Ministerio de Educación Nacional de Colombia, el número de estudiantes por computador en los establecimientos educativos gubernamentales ha disminuido en tan sólo siete años de vigencia del programa a menos del $50 \%$ de los indicadores iniciales. Por otra parte, la conexión a internet en las sedes educativas 
oficiales tiene como meta alcanzar para 2018 el 90\% de cobertura, en el periodo de abril de 2018 esta meta se tradujo en 13.222 instituciones de educación con conexión disponible de internet con fines académicos.

(Datostomados de: https://www.mineducacion.gov.co/1759/w3-article-348154.html)

\section{Número de Estudiantes promedio por computador}

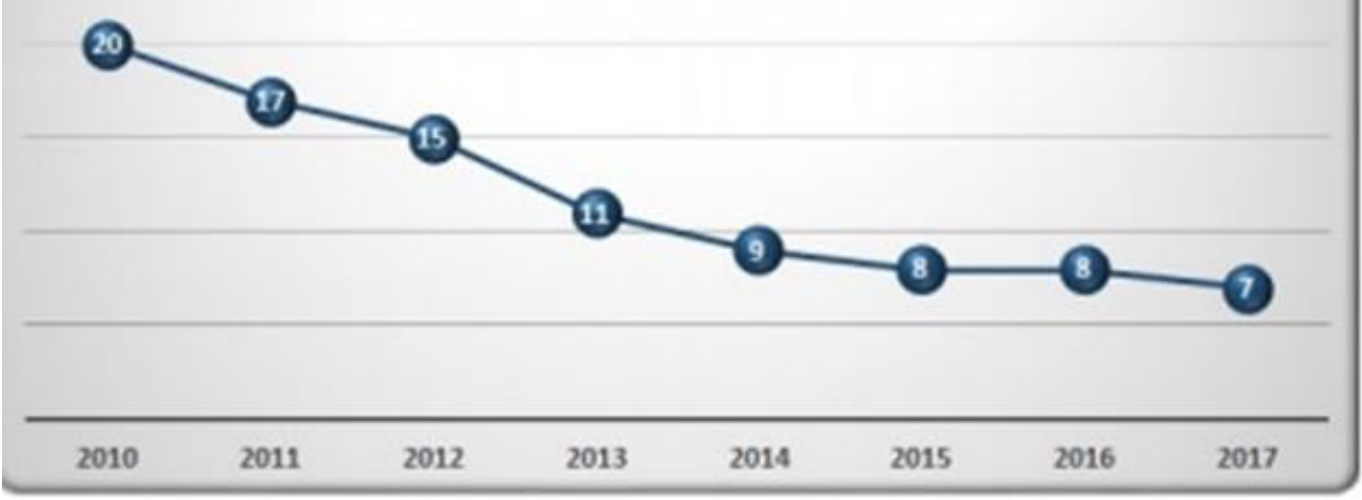

Tomado de https://www.mineducacion.gov.co/1759/articles-354999_recurso_1.jpg

En este contexto de globalización y apertura a las nuevas tecnologías los procesos educativos y pedagógicos del aula encuentra posibilidades, retos, pero también amenazas a las viejas estructuras y paradigmas que deben evolucionar a la velocidad misma de la red.

\section{El sistema educativo colombiano y la enseñanza de la segunda lengua}

En la Constitución Política, Colombia se define a sí misma como un "Estado Social de Derecho", garante de los derechos fundamentales, entre ellos a la educación como se consagra en los artículos 67 y 68 de la Carta Magna. Así mismo, se enuncia que este derecho será vigilado y regulado por las entidades correspondientes. El ente rector de las políticas educativas y quien traza los lineamientos generales para la prestación del servicio educativo, es el Ministerio de Educación Nacional (MEN), cuya normatividad está enmarcada en la Ley General de Educación, ley 115 del 8 de febrero de 1994. Para el caso particular de la enseñanza de las lenguas, ésta refiere a la "adquisición de elementos de conversación y de lectura al menos en una lengua extranjera" (Artículo 21). Para dar cumplimento a esta Ley, el gobierno se vale de los Planes Nacionales de Desarrollo, siendo "Todos por un nuevo país" el actual Plan Nacional de Desarrollo 2014-2018, encaminado a mejorar la calidad y cobertura educativa en el país. Como eje articulador para su cumplimento, se promulga el Programa Nacional Inglés (2015-2025) que, junto con el Plan Nacional de Bilingüismo, "inglés como lengua extranjera: Una estrategia para la competitividad (2004- 2019)" buscan ejecutar proyectos estratégicos para la competitividad: Uso de medios y nuevas tecnologías, competencias laborales y bilingüismo como el mejoramiento de las competencias comunicativas en inglés como lengua extranjera en todos los sectores educativos. 
Para la estandarización de todo este proceso, el Gobierno Nacional expide el decreto 3870 de 2006 en donde se adopta el Marco Común Europeo de Referencia para las Lenguas como el sistema de referencia para los procesos de aprendizaje, enseñanza y evaluación adelantados en Colombia. De esta manera, las metas específicas del Programa Nacional de Bilingüismo para las instituciones de educación superior proponen el alcance de un nivel B2 en los estudiantes graduados de sus programas académicos. Esto evidencia el interés puntual del Gobierno Nacional por adelantar acciones que mejoren la cobertura del servicio dirigido a la formación en lengua extranjera en la educación del país.

Ahora bien, con el fin de conocer el nivel de los estudiantes de todo el país y en los diferentes niveles del sistema educativo, Colombia ha figurado en el Índice Mundial de Competitividad del inglés (English Proficiency Index) con resultados que no concuerdan con el andamiaje legal y normativo anteriormente presentado. En uno de los últimos reportes publicados, Colombia aparece en el puesto 51 en un total de 80 países. El nivel de clasificación nos ubica en la posición 11 de 15 países latinoamericanos sin que haya mucha variación en relación a los reportes anteriores:

Tabla 1. Análisis de las tendencias del EF EPI

\begin{tabular}{l|lllll|l}
2011 & 2012 & 2013 & 2014 & 2015 & 2016 & 2017 \\
\hline Puesto & Puesto & Puesto & Puesto & Puesto & Puesto & Puesto \\
$41 / 44$ & $50 / 54$ & $46 / / 60$ & $42 / 63$ & $57 / 70$ & $49 / 72$ & $51 / 80$ \\
\hline Muy bajo & Muy bajo & Muy bajo & Bajo & Muy bajo & Muy bajo & Bajo
\end{tabular}

Tomado de: https://www.ef.com.co/epi/regions/latin-america/colombia/

A pesar de no reconocer oficialmente los resultados publicados por el Índice Mundial de Competitividad en el inglés, el Ministerio de Educación Nacional se ve en aprietos al momento de verificar el cumplimento de las metas propuestas en los programas mencionados. El Programa Colombia Bilingüe espera subir para el año 2018 el nivel Pre Intermedio (B1 de acuerdo al Marco Común Europeo de Referencia para las Lenguas MCERL) del 2\% al 8\% y del 7\% al 35\% de los estudiantes en nivel Básico A2 - MCERL; sin embargo, los resultados brindados por las pruebas Saber 11 sitúan tal alcance en un 4,75\% a 2017 en nivel B1 dejando tan sólo el periodo de un año para alcanzar el 3,25\% restante para sólo un periodo electivo. Aunque es innegable reconocer los esfuerzos del Gobierno en avanzar en estos índices de competitividad (se pasó de un 1\% de estudiantes en B1 en 2014) y en el efecto que los planes propuestos han tenido en el desarrollo de las competencias necesarias para los estudiantes alcanzar niveles de desempeño mayores, es necesario avanzar más e incorporar nuevas estrategias que impulsen en mayor grado los logros alcanzados.

\section{El uso de dispositivos móviles en la enseñanza de segunda lengua en la Universidad Santo Tomás - "no me dejes en visto"}

Este es el panorama. Estas son las cifras. ¿Es esta la realidad? Como docentes de una institución de educación superior nos encontramos con oportunidades más desafiantes. La Universidad Santo Tomás de Bogotá es una Institución de Educación Superior (IES) de 
Colombia y está avalada por el Ministerio de Educación Nacional con Acreditación de Alta Calidad Multicampus. La formación en lengua extranjera ha sido un elemento importante en la consolidación de programas académicos de alta calidad por lo que en 2014 la Universidad promulgó su Política de Adquisición de competencia comunicativa en lengua extranjera a través del Acuerdo 46 articulada a su vez a los Planes de Desarrollo 2012 - 2015 y Plan Integral Multicampus (PIM) 2016 - 2027. Como parte de la implementación del Acuerdo 46 se elaboró un plan diagnóstico de competencia en inglés a toda la comunidad académica, poniendo especial énfasis en los estudiantes que ingresan por primera vez a la universidad. Como resultado de esta exploración inicial, se consolidó la aplicación de la prueba diagnóstica Oxford Online Placement Test-OOPT, cuyos resultados son el insumo para la clasificación de los estudiantes que ingresan provenientes de los establecimientos de educación media. Los resultados de los últimos cinco años se contraponen con la meta del Ministerio de Educación Nacional ya que como se puede evidenciar los estudiantes que terminan el ciclo de educación básica no completan en su totalidad las metas propuestas por el Plan de Bilingüismo.

En las metas del Ministerio de Educación se plantea que las Instituciones de educación básica y media deben entregar a los estudiantes en nivel $\mathrm{B} 1$ para que las Universidades puedan completar la meta de alcanzar un nivel B2 o superior al finalizar sus estudios profesionales; sin embargo, el $77 \%$ de los estudiantes que ingresan a la Universidad Santo Tomás y quienes presentan la prueba diagnóstica se ubican en un nivel A1 y sólo el 7\% se ubica entre los niveles B1 y B2.

Ahora bien, ¿Están dadas las condiciones para brindar la formación en competencias comunicativas en ingles acorde a los parámetros nacionales e institucionales en los estudiantes de la USTA? ¿Qué tipo de estrategias se pueden implementar en el aula de clase de manera que se fortalezca el currículo, se brinden mayores oportunidades de mejoramiento y se tome ventaja de los conocimientos de los estudiantes? ¿Cómo se puede aprovechar el perfil del estudiante de la Universidad Santo Tomás para que él mismo sea el protagonista de su aprendizaje? ¿Cómo se puede hacer efectivo el aprendizaje de una lengua extranjera en una sociedad globalizada?

Estos son algunos de los interrogantes que dieron inicio a la experiencia "No me dejes en visto" de la mano de dos docentes de la Universidad Santo Tomás quienes inician el diseño e implementación de una experiencia pedagógica a inicios de 2018 con el propósito de involucrar las herramientas digitales, disponibles para los estudiantes de pregrado de los grupos a su cargo en la Universidad Santo Tomás, en el desarrollo de las competencias comunicativas necesarias para dar respuesta a los requerimientos institucionales y nacionales con respecto a los lineamientos de formación en lengua extranjera.

La población en la que se han trabajado las estrategias de inclusión de las TIC y en particular el uso de dispositivos móviles es mayoritariamente perteneciente a los estratos socio económicos 3-4 lo que implica que los estudiantes participantes tienen un manejo medio-alto de las tecnologías de la comunicación y tienen acceso a laptop o teléfono móvil inteligente 
durante las sesiones de clase. De igual manera, la Universidad Santo Tomás provee de la facilidad de conectividad a internet en sus edificios lo que promueve constantemente el uso de la red por parte de la comunidad educativa.

El objetivo primordial de la experiencia investigativa es proporcionar estrategias que promuevan el desarrollo de las competencias comunicativas en los estudiantes del Instituto de Lenguas Fray Bernardo de Lugo O.P. (ente encargado de la formación en lenguas extranjeras en la Universidad Santo Tomás) mediante la inclusión del teléfono móvil como herramienta didáctica que fomenta la formación de competencias del siglo XXI no sólo en el ámbito particular de la lengua extranjera sino también en el desarrollo de las destrezas y habilidades enmarcadas en la sociedad de la información y conocimiento.

A partir de la exploración del paradigma del procesamiento de la información, el grupo de investigadores encontró en el enfoque cognitivo el camino para proponer el desarrollo de la experiencia pedagógica. El enfoque cognitivo se interesa por el estudio de la representación mental, por lo que busca describir y explicar la naturaleza de las representaciones mentales y su papel en la conducta humana la cual no es regulada por el medio externo, sino por las representaciones elaboradas por el sujeto. Se rompe de esta manera con la concepción de sujeto cognoscente del conductismo pues éste ya no es visto como una tabula rasa, sino que éste es un ente activo que organiza las representaciones, de modo que, al menos en este enfoque, nuestro paradigma se encuentra dentro del postulado constructivista para dar cuenta de su interés por describir y explicar los mecanismos de la mente humana, es decir, dar cuenta del procesamiento de la información. Así pues, en el marco del cognitivismo, los investigadores buscan empoderar a los estudiantes en su proceso de adquisición de la lengua extranjera permitiéndoles, a partir de su propia experiencia, ser gestores y mediadores de su aprendizaje.

Ahora bien, revisemos cómo este paradigma tiene aplicaciones en el campo educativo y cómo éstas han sido usadas. Dos autores que han reflexionado y pensado en esta relación han sido J. Bruner y D.PD. Ausubel. El primero se destacó por ser uno de los psicólogos cognitivos de mayor renombre en Estados Unidos gracias a su teoría del aprendizaje por descubrimiento y el currículo para pensar. Por su parte, Ausubel es reconocido gracias a su teoría del aprendizaje significativo y de la asimilación. Estas concepciones tienen puntos en común en cuanto a la manera en que se concibe el proceso de enseñanza -aprendizaje y el papel de sus actores. Para estos enfoques, la educación debe orientarse en lograr el desarrollo de habilidades de aprendizaje, por lo que la concepción de enseñanza va más allá de la simple transferencia de saberes. Así, se da importancia al desarrollo de habilidades y estrategias que permitan aplicar los conocimientos adquiridos en diferentes situaciones. Hay un marcado interés por el desarrollo del potencial del estudiante, de modo que éste sepa no sólo cómo aprender, sino también cómo solucionar problemas. Precisamente es allí, en donde la experiencia "No me dejes en visto" cobra relevancia: se busca traspasar la barrera de la exposición gramatical como medio para el aprendizaje de la lengua mediante el desarrollo de habilidades de aprendizaje en los estudiantes que los lleven a utilizar los dispositivos móviles

Revista Revoluciones -52- Vol. 2, Nº 2 (2020), pp. 45-59

Esta obra está bajo una licencia internacional Creative Commons Atribución 4.0. 
de los que hacen uso cada día como las herramientas que les pueden llevar a trasladar el conocimiento adquirido de la lengua en situaciones reales, contextos auténticos en los que la lengua que aprenden significa realmente algo: la oportunidad de conectarse con el mundo y participar activamente como sujetos participantes de una comunidad global.

En este sentido, los objetivos de la experiencia pedagógica se encaminan al desarrollo del pensamiento, proceso que puede ser alcanzado en la conocida taxonomía de Bloom. Recordémosla rápidamente: Se inicia con el conocimiento (base de retención de la información provista principalmente mediante dispositivos móviles y herramientas digitales), luego se pasa a la comprensión (se entiende la información), el análisis que supone utilizar la información, seguidamente de la síntesis (se analiza la información en sus partes constitutivas - se encuentra la significación de ese conocimiento y su propósito) y finalmente está la evaluación, donde podemos ya emitir juicios sobre el material enseñado.

Para el propósito de la experiencia "No me dejes en visto", el alumno se concibe como un sujeto activo procesador de información, el cual posee unos esquemas y estrategias de solución de problemas que deben ser desarrollados, de modo que cobra sentido la importancia de la acción frente al conocimiento y sus habilidades. En este mismo sentido, la labor del maestro está encaminada al diseño de experiencias didácticas que logren el desarrollo del aprendizaje significativo y de habilidades cognitivas y meta cognitivas en los estudiantes.

Con todo lo anterior, se puede decir que la concepción de creación del conocimiento, del sujeto como parte activa y protagonista de dicho proceso, y los elementos constitutivos del cognitivismo, permiten tener una mirada más abarcadora del proceso de enseñanza aprendizaje con el fin de aportar cada vez más al desarrollo de sujetos con pensamiento crítico y reflexivo, en capacidad de dar solución a las demandas de sus contextos locales y globales lo cual va más allá del alcance de un nivel de inglés y asegura la consecución de metas aún mayores: formar a los ciudadanos de la sociedad globalizada.

“iNo me dejes en visto!" inició con una exploración acerca de los usos dados por los estudiantes al teléfono móvil, los tiempos dedicados a tales actividades y las aplicaciones más comúnmente utilizadas. Esta pesquisa inicial brindó un panorama del uso de dispositivos móviles por parte de los 258 estudiantes participantes en los periodos 2018-2019:

Datos obtenidos a partir de la aplicación de encuestas. Periodos 2018-2019

Al indagar si los dispositivos móviles podrían ser usados con fines académicos, los datos obtenidos dan cuenta de un uso mayoritario para la práctica de la habilidad de escucha (mediante la exposición a material en inglés y en menor grado, el acceso a información en la lengua objetivo.

Datos obtenidos a partir de la aplicación de encuestas. Periodos 2018-2019

Revista Revoluciones -53- Vol. 2, No 2 (2020), pp. 45-59

Esta obra está bajo una licencia internacional Creative Commons Atribución 4.o. 
A partir de los resultados obtenidos y con la exploración de aplicaciones diseñadas para ser utilizadas en el ámbito educativo, se lograron vincular al desarrollo del currículo diferentes estrategias que permitieron involucrar el uso del teléfono móvil en el aula, éstas buscan fomentar el aprendizaje significativo y brindar la oportunidad -a docentes y estudiantes- de explorar herramientas diferentes a las tradicionales usadas en el aula (tablero, libro de texto, computador, diapositivas) involucrando las experiencias personales en el manejo de las TIC.

Antes de presentar las herramientas utilizadas en la experiencia pedagógica, es importante aclarar que el equipo investigador aún está desarrollando la investigación acerca del impacto de la inclusión de estrategias de inclusión de dispositivos móviles en el aula. El hecho de que cada semestre se cambie de estudiantes hace que la medición de los resultados a largo plazo se dificulte, aunque a corto plazo; es decir durante el período académico de un semestre, se ha logrado evidenciar ciertos avances que serán referenciados más adelante en el documento.

Con el objetivo de usar los dispositivos móviles disponibles en el aula, los docentes investigadores involucraron el desarrollo de la lección a través de algunas de las aplicaciones que se glosan a continuación:

- Nearpod: Herramienta en línea que permite la presentación de lecciones interactivas. Los estudiantes tienen la oportunidad, entre otras características disponibles en la aplicación, de presentar comentarios o imágenes creadas por ellos mismos, así como responder encuestas en tiempo real o realizar visitas virtuales en diferentes lugares del mundo. Todo el desarrollo de la lección es seguido por el estudiante a través de su teléfono.

No requiere registro previo por parte de los estudiantes. El docente puede buscar en la biblioteca material disponible acorde con sus necesidades y también puede crear sus propias presentaciones. Para el acceso a características PRO, es necesario adquirir la membresía. Disponible en www.nearpod.com

\section{Servicios disponibles en el servidor Google:}

- Google docs: Servicio de creación y alojamiento de archivos que pueden ser editados por varios usuarios al mismo tiempo. Permite establecer redes de trabajo colaborativo entre los estudiantes mediante la asignación de roles y responsabilidades. A través de la aplicación disponible para dispositivos móviles, los estudiantes pueden acceder a hojas de cálculo, documentos, formularios, presentaciones, etc. y editarlos en tiempo real. Se requiere iniciar sesión con cuenta de correo GMAIL, en el caso de la Universidad Santo Tomás, con la cuenta de correo institucional. Disponible en www.docs.google.com

- Meet: Servicio de video llamada que permite brindar a los estudiantes espacios no convencionales para la práctica de la competencia oral desde su dispositivo móvil. A través de la plataforma se pueden realizar sesiones de tutoría para estudiantes con

Revista Revoluciones -54- Vol. 2, No 2 (2020), pp. 45-59 
necesidades particulares, así como ejercicios de práctica oral no limitados a un espacio físico. El docente puede realizar video llamadas hasta con 25 personas, aunque en la experiencia de los investigadores, un grupo de máximo 6 estudiantes puede aprovechar al máximo el espacio de encuentro virtual. Se puede acceder a la aplicación mediante el registro con el correo GMAIL. El equipo investigador recomienda la plataforma Meet sobre otros servicios similares como Whatsapp o Skype pues no necesita la creación de cuentas adicionales a la de correo, en el caso de la USTA correo institucional, o el manejo de información personal como el número de teléfono, en el caso de Whatsapp. Disponible en: www.meet.google.com

- Youtube: Sitio web que permite la oportunidad de exposición a muestras reales de la lengua a través de una extensa biblioteca. Los estudiantes pueden buscar contenido acorde a sus gustos o preferencias en términos de campo de estudio o motivo de práctica. La exposición continua favorece el desarrollo de la habilidad de escucha y pronunciación. Adicionalmente puede usarse para la publicación de material producido por los mismos estudiantes y disponible para que sea revisado por otros estudiantes. Para la búsqueda o publicación de material en ésta plataforma se requiere una dirección clara por parte del docente si va a ser utilizada como apoyo a la lección ya que dada la cantidad de videos publicados puede convertirse en un riesgo sobre todo en cuanto a la exposición a material con contenido sexual o violento. Disponible en: www.youtube.com

Recomendamos los siguientes canales:

https://www.youtube.com/user/BritishCouncilLE

https://www.youtube.com/user/bbclearningenglish/featured

https://www.youtube.com/user/duncaninchina/videos

https://www.youtube.com/user/TEDtalksDirector

- Wordreference: Diccionario en línea. Su uso permite la creación de hábitos de uso del diccionario para la búsqueda de vocabulario desconocido y busca limitar el uso de traductores. Adicionalmente permite la identificación de categorías gramaticales lo que lleva a un aprendizaje sistemático de la lengua. Disponible en: www.wordreference.com

\section{Plataformas para la práctica lúdica:}

- Kahoot: Plataforma gratuita en línea para la creación y administración de juegos tipo cuestionario. Brinda la oportunidad de practica de manera lúdica aspectos puntuales de la lección (estructuras gramaticales, vocabulario, control de lectura, etc.) mediante juegos de competencia de organizar la estructura o de selección múltiple. Los estudiantes participan registrándose con sus teléfonos celulares. El docente puede acceder a la biblioteca disponible en la plataforma o puede crear su propio juego acorde a sus necesidades. No necesita registro previo para los estudiantes, aunque si para el docente. Disponible en: www.kahoot.com

Revista Revoluciones -55- Vol. 2, Nº 2 (2020), pp. 45-59

Esta obra está bajo una licencia internacional Creative Commons Atribución 4.0. 
- Quizlet: Plataforma en línea que permite la práctica individual mediante pruebas cortas y tarjetas educativas con vocabulario a ser estudiado entre otros siendo un apoyo para la asignación de trabajo autónomo a los estudiantes; como herramienta durante el desarrollo de la lección en la clase, permite la creación de grupos de trabajo propiciando la interacción con otros compañeros mientras se establecen reglas de trabajo colaborativo para la resolución de problemas relacionados con el propósito de la lección. Es una herramienta gratuita que no requiere registro de los estudiantes, pero si del docente quien puede explorar la biblioteca con las actividades disponibles o crear las propias. Disponible en: www.quizlet.com

\section{Redes sociales dirigidas al aprendizaje}

En cuanto a redes sociales, la variedad es mucha dependiendo del perfil de los estudiantes; por ejemplo, los estudiantes de Diseño prefieren Pinterest o Instagram por el contenido visual al que pueden acceder mientras que para estudiantes con otras necesidades Facebook, Twitter o Snapchat pueden brindar oportunidades valiosas de exposición y práctica de la lengua extranjera. Dentro de la experiencia pedagógica "No me dejes en visto" el uso de las redes sociales se ha dado en las siguientes modalidades:

- Participación en grupos: Favorece la producción escrita de textos cortos dirigidos al debate o presentación de puntos de vista frente a un tema de discusión trabajado en clase.

- Descripción de imágenes o situaciones en contextos reales: Desde la consulta del clima a noticias del día que pueden ser consultadas en el momento de la lección hasta la discusión en torno a comentarios en la red, esta estrategia permite el desarrollo de pensamiento crítico en los estudiantes mientras se concientizan de su entorno y proponen soluciones que pueden ser presentadas en comentarios cortos o videos publicados en la red social acordada.

Al tener la herramienta del teléfono móvil en sus manos, los estudiantes son capaces de resolver problemas que surgen durante el desarrollo de la lección; desde el simple hecho de buscar el significado de un palabra desconocida hasta dar su opinión crítica de un evento leído en un portal de noticias, los estudiantes manifiestan una apropiación de la herramienta que deja de ser un elemento ajeno a la clase y pasa a convertirse en un elemento útil dentro del proceso de desarrollo de competencias comunicativas, autonomía y empoderamiento de su aprendizaje. El equipo investigador ha encontrado que a través de la inclusión del teléfono celular se crean nuevos espacios de aprendizaje que al inicio son mediados por el docente pero que van evolucionando hasta ser oportunidades de participación en la sociedad del conocimiento que no se limitan a un tiempo o un espacio, sino que son permanente aprovechados por los estudiantes que ahora se apropian de su rol como participantes conectados con el mundo. 


\section{CONCLUSIONES}

Como resultado de la implementación de la experiencia "No me dejes en visto", son claras las implicaciones del uso de teléfonos inteligentes como herramienta didáctica que posibilita el aprendizaje significativo enmarcado en el cognitivismo en los estudiantes y, a la vez, supone un reto para que los docentes abandonemos las estrategias de enseñanza tradicionales, y vinculemos nuevas estrategias para propiciar ejercicios de pensamiento de orden superior, para nuestro caso, en el aprendizaje de una lengua extranjera.

Dentro de las oportunidades que surgen a partir de la implementación de las estrategias presentadas, se encontró que una de ellas es la calidad de la conectividad a internet en el aula. A pesar de ser una institución con acreditación de alta calidad, la conexión a la red inalámbrica en la USTA traía consigo la incertidumbre de saber si podría realizarse la actividad propuesta. Esta particularidad puede derivar en que los estudiantes se distraigan del propósito inicial de la lección. Otra oportunidad yace en el análisis y posible uso de aplicaciones de software -apps- dirigidas al aprendizaje de lenguas como herramientas de apoyo a los procesos de aula. La puerta está abierta y el camino está delante de nosotros.

Como etapa siguiente a la implementación de esta estrategia, el equipo de investigadores busca establecer posibles relaciones entre los retos de la adquisición de competencia en lengua extranjera y el modelo de aprendizaje móvil o mLearning como oportunidad para los estudiantes de alcanzar mayor y mejor desempeño en la lengua y para los docentes como un reto de innovación dirigida hacia la construcción de un modelo educativo acorde con las necesidades de la generación de millenials, nativos digitales; en otras palabras, nuestros estudiantes.

\section{BIBLIOGRAFÍA}

Ander Egg, E., (2000) El proceso de Globalización en la Cultura. Cuadernos Patrimonio Turístico y Cultural, N. 13, pp. 144-164.

Ahmad, K., Armarego, J. and Sudweeks, F. (2013). Literature review on the feasibility of mobile-assisted language learning (MALL) in developing vocabulary skills among non-English speaking migrant and refugee women. International Conference on Research and Innovation in Information Systems, ICRIIS. pp. 336-341. doi: 10.1109/ICRIIS.2013.6716732

Ally, M. \& Prieto-Blázquez, J. (2014). What is the future of mobile learning in education? Mobile Learning Applications in Higher Education [Special Section]. Revista de Universidad y Sociedad del Conocimiento (RUSC). Vol. 11, No 1. pp. 142-151. doi http://dx.doi.org/10.7238/rusc.v11i1.2033.

Botia, A. (2002). Case of study as biography-narrative report. Revista Arbor, ciencia, pensamiento y cultura. Vol. 171, Issue 675, pp. 559-578. Recuperado de: http://arbor.revistas.csic.es/index.php/arbor/article/viewFile/1046/1053 
Chen, C. M. \& S.-H. Hsu. (2008). "Personalized Intelligent Mobile Learning System for Supporting Effective English Learning”. Educational Technology \& Society, 11 (3), pp. 153-180.

Claro, M., CEPAL. (2010). La Incorporación de Tecnologías Digitales en Educación. Modelos de Identificación de Buenas Prácticas. Santiago de Chile, Chile: Naciones Unidas.

Del Campo Cañizares, E. (2014). M-Learning y aprendizaje informal en la educación superior mediante dispositivos móviles. Revista Historia Y Comunicación Social, 18, pp. 231242. https://doi.org/10.5209/rev_HICS.2013.v18.44239

EF.COM.CO (2018). Inicio - EF EPI. English Proficiency Index. [en línea] Disponible en: https://www.ef.com.co/epi/downloads/

Elaish M., Shuib L., Abdul Ghani A., Yadegaridehkordi E. and Alaa M. (2017). Mobile Learning for English Language Acquisition: Taxonomy, Challenges, and Recommendations. IEEE Access, vol. 5, pp. 19033-19047, doi: 10.1109/ACCESS.2017.2749541

Flétscher, L., Morales, A. (2007) Modelo de desarrollo de servicios m-learning, una propuesta desde la concepción del servicio hacia la pedagogía. Revista Virtual Universidad Católica del Norte. Núm.22. 2007 pp. 1-22

Gardner, H., \& Davis, K. (2014). La generación APP. Cómo los jóvenes gestionan su identidad, su privacidad y su imaginación en el mundo digital. Bogotá: Planeta.

Gómez, C. V., \& Marta-Lazo, C. (2015). Modelo De Integración Educomunicativa de "Apps" Móviles Para la Enseñanza Y Aprendizaje. Pixel-Bit, Revista de Medios y Educación, (46), 137-153. https://doi-org.craiustadigital.usantotomas.edu.co/10.12795/pixelbit.2015.i46.09

Godwin-Jones, R. (2011). Emerging technologies: Mobile apps for language learning. Language Learning and Technology, 15(2). pp. 2-11 http://dx.doi.org/10125/44244

Godwin-Jones, R. (2018). Using mobile devices in the language classroom: Part of the Cambridge Papers in ELT series. Cambridge University Press

Hernández, G. (1997). Caracterización del Modelo Cognitivo. México: ILCE - OEA. Ley 115 (1994). Por la cual se expide la ley general de educación, en http://www.mineducacion.gov.co/1621/articles-85906_archivo_pdf.pdf

Hernández. S. (2014). Metodología de la investigación. Sexta edición. México: McGraw-Hill Interamericana.

Huang, C. and P. Sun. (2010) "Using mobile technologies to support mobile multimedia English listening exercises in daily life". In: The International Conference on Computer and Network Technologies in Education (CNTE 2010), At: http://cnte2010.cs.nhcue.edu.tw/

Ibañez, A. y Traxler J. (2016). MALL-Based MOOCs for Language Teachers: Challenges and opportunities. Porta Linguarum. Revista Internacional de Didáctica de las Lenguas Extranjeras. Monográfico I. 2016. 73-85

Klopfer, E., Squire, K., \& Jenkins, H. (2002). Environmental detectives PDAs as a Window into a virtual simulated world. IEEE International Workshop on Wireless and Mobile Technologies in Education, 95-98. https://doi:10.1109/WMTE.2002.1039227 
Lozano, R. (2011) De las TIC a las TAC: tecnologías del aprendizaje y del conocimiento. Anuario ThinkEPI, 2011, v. 5, pp. 45-47. Recuperado de: https://recyt.fecyt.es//index.php/ThinkEPI/article/view/30465

MEN, (2009) Organización del Sistema Educativo, [en línea] Disponible en: https://www.mineducacion.gov.co/1759/articles- 205294_archivo_pdf.pdf

MEN, (2018). Inicio - Ministerio de Educación Nacional de Colombia. [en línea] Disponible en: http://www.mineducacion.gov.co/1621/article-107522.html

MEN. (2016). La Innovación Educativa en Colombia -Buenas Prácticas para la Innovación y las TIC en Educación. Bogotá, Colombia. Recuperado de https://aprende.colombiaaprende.edu.co/ckfinder/userfiles/files/Libro\%2oInnov acion\%20MEN\%20-\%20V2.pdf

Puchmüller, A. y Puebla, M. (2014). TIC en Educación Superior: usos e implicancias en dos carreras de instituciones argentinas. Revista Encuentros, 12(2). doi: 10.15665/re.v12i2.266

Rezaei A., Neo, M. and Pesaranghader A. (2013) Effectiveness of using English vocabulary mobile applications on ESL's Learning performance. Proceedings - 2013. International Conference on Informatics and Creative Multimedia. 2013, pp. 114-118.

Rodríguez, L., Vallejo G., Proaño, F., Romero, H., Solís, L., y Erazo, J. (2017). Diseño de una metodología m-learning para el aprendizaje del idioma inglés. Revista Boletín Redipe, 6(6), $\quad 35 \quad$ - $\quad 48 . \quad$ Recuperado a de https://revista.redipe.org/index.php/1/article/view/241

Rosell-Aguilar, F. (2017). State of the app: A taxonomy and framework for evaluating language learning mobile applications. CALICO Journal 34(2) pp. 243258. https://doi.org/10.1558/cj.27623

Salguero Cubides, J., (2011) Globalización. Economía y regiones de Colombia. Sociedad geográfica de Colombia.

Seiz Ortiz, Rafael. (2015) Aprendizaje móvil de lenguas basado en criterios pedagógicos. @ @ic. revista d'innovació educativa Universidad de Valencia. Disponible en http://www.redalyc.org/articulo.oa?id=349543461011

Taylor, S. y Bogdan, R. (1986). Introducción a los métodos cualitativos de investigación. Buenos Aires, Argentina: PAIDOS

Torrente, P. (2012) E-Learning para la enseñanza de lenguas extranjeras: factores de éxitos y desafíos., Revista Teckne, 10 (2) Págs. 54-58.

UNESCO (2013). Directrices para las políticas de aprendizaje móvil. París, UNESCO. Recuperado de https://unesdoc.unesco.org/ark:/48223/pfoooo219662

Universidad Santo Tomás. (2015). Documento Marco Lenguas Extranjeras. Bogotá, Colombia: Ediciones USTA.

Universidad Santo Tomás (2015). Lineamientos para el diseño y la actualización curricular. Bogotá: Ediciones USTA.

Universidad Santo Tomás. (2016). Plan Integral Multicampus. Bogotá, Colombia: Ediciones USTA. 